\title{
De la insuficiencia a la falla intestinal, un camino de doble sentido que cruza con la malnutrición
}

\author{
From insufficiency to intestinal failure, a two-way road that crosses \\ malnutrition
}

\author{
Da disfunção à insuficiência intestinal, uma via de mão dupla que cruza com a desnutrição
}

Diana Trejos-Gallego ${ }^{1 *}$, Clara Helena González-Correa², William Narváez-Solarte²

Recibido: 26 de enero de 2021. Aceptado para publicación: 11 de marzo de 2021.

Publicado en línea: 23 de marzo de 2021.

https://doi.org/10.35454/rncm.v4n3.261

\section{Resumen}

Introducción: actualmente, la insuficiencia y la falla intestinal están presentes en cerca del $60 \%$ de los pacientes en unidades de cuidado intensivo en el mundo, siendo más frecuente la presencia de insuficiencia. El objetivo de esta revisión es presentar la evidencia actual sobre la relación de la insuficiencia, la falla intestinal y su influencia en el desarrollo de la malnutrición hospitalaria.

Métodos: se realizó una búsqueda en Medline y PubMed de las publicaciones que incluyeran las palabras clave "insuficiencia intestinal", "falla intestinal", "soporte nutricional", "malnutrición hospitalaria", de artículos en inglés y español.

Resultados: la presencia de falla e insuficiencia intestinal aumenta la probabilidad de malnutrición en el paciente en estado crítico debido a que la absorción de nutrientes se ve disminuida $y$, por ende, lo hace más vulnerable a desarrollar una mayor pérdida de peso, lo que aumenta su morbimortalidad. Además, la estancia hospitalaria de los pacientes es más prolongada, y genera altos costos al sistema de salud. Esto se debe a que frecuentemente se presentan infecciones y complicaciones asociadas con la desnutrición y la pérdida de productividad, lo que disminuye la calidad de vida, con efectos negativos sobre el entorno social.

Conclusiones: la insuficiencia y falla intestinal son situaciones que pueden

\section{Abstract}

Introduction: Intestinal insufficiency and intestinal failure are currently found in close to $60 \%$ of patients in intensive care units in the world, the presence of insufficiency being more frequent. The objective of this review is to present the current evidence on the relationship between intestinal insufficiency and failure and their influence on the development of hospital malnutrition.

Methods: A search was carried out in Medline and PubMed for publications that included the keywords "intestinal insufficiency", "intestinal failure", "nutritional support", "hospital malnutrition", in articles in English and Spanish.

Results: The presence of intestinal failure and insufficiency increases the probability of malnutrition in critically ill patients because of reduced nutrient absorption, making them more vulnerable to greater weight loss, and increasing morbidity and mortality. In addition, hospital stay is longer, resulting in high costs for the health system associated with frequent malnutrition-related infections and complications. Moreover, there are repercussions in term of lost productivity, lower quality of life and negative effects on the social environment.

Conclusions: Intestinal insufficiency and failure can be a bidirectional situation. Differences in the classification of intestinal failure according to the various associations affect the management of patients.

\section{Resumo}

Introdução: a insuficiência e a falência intestinal estão presentes atualmente em cerca de $60 \%$ dos pacientes internados em unidades de terapia intensiva no mundo, sendo a presença de insuficiência mais frequente. $\mathrm{O}$ objetivo desta revisão é apresentar as evidências atuais sobre a relação entre insuficiência, falência intestinal e sua influência no desenvolvimento da desnutrição hospitalar.

Métodos: foi realizada uma pesquisa bibliográfica no Medline e no Pubmed de publicações com as palavras-chave "intestinal insufficiency", "intestinal failure", "nutricional support", "hospital desnutrition", de artigos em inglês e espanhol.

Resultados: a presença de insuficiência e insuficiência intestinal aumenta a probabilidade de desnutrição em pacientes críticos, pois a absorção de nutrientes é diminuída e, portanto, os torna mais vulneráveis a desenvolver maior perda de peso, aumentando sua morbimortalidade. Além disso, o tempo de internação dos pacientes é maior e gera custos elevados para o sistema de saúde. Isso se deve às infecções e complicações frequentes associadas à desnutrição e perda de produtividade, diminuindo a qualidade de vida, com reflexos negativos no meio social.

Conclusões: a insuficiência e falência intestina são situações que podem ser geradas de forma bidirecional. As diferenças na classificação da falência intestinal de 
generarse de manera bidireccional. Las diferencias en la clasificación de la falla intestinal según las diversas asociaciones afectan el manejo de los pacientes.

Palabras clave: insuficiencia intestinal, falla intestinal, soporte nutricional, malnutrición hospitalaria.
Keywords: Intestinal Dysfunction; Intestinal Failure; Nutritional Support; Hospital Malnutrition. acordo com as várias associações afetam o manejo dos pacientes.

Palavras clave: falência intestina, insuficiência intestinal, suporte nutricional, desnutrição hospitalar.
Doctorado en Ciencias Biomédicas, Universidad de Caldas, Manizales, Caldas, Colombia.
2 Grupo de Investigaciones en Nutrición, Metabolismo y Salud. Universidad de Caldas, Manizales, Caldas, Colombia.

\section{DECLARACIÓN DE RELEVANCIA CLÍNICA}

Es necesario visibilizar la insuficiencia y la falla intestinal como situaciones bidireccionales que se presentan en el paciente crítico y que, a su vez, son determinantes para el desarrollo de la desnutrición, esto para poder ajustar las prácticas actuales a un enfoque más simple e integral, que permita el abordaje desde el punto de vista médico y nutricional.

\section{INTRODUCCIÓN}

Según la European Society for Clinical Nutrition and Metabolism (ESPEN), la falla intestinal es la reducción de la función intestinal por debajo del mínimo necesario para la absorción de macronutrientes o agua y electrólitos, de modo que se requiere una suplementación intravenosa para mantener la salud o el crecimiento ${ }^{(1)}$. En la medicina crítica, hablar de falla intestinal genera confusión, debido a que esta condición clínica no se clasifica como una falla orgánica, como sí ocurre para los sistemas respiratorio, renal, circulatorio y hepático ${ }^{(2)}$. Esta situación dificulta el diagnóstico y el tratamiento oportunos de la enfermedad, ya que no existe una clasificación internacional estándar de la misma, por lo que la interpretación e identificación de los signos clínicos que presenta el paciente queda a criterio del equipo encargado del soporte nutricional ${ }^{(1)}$. Por esta razón, la ESPEN ha tratado de definir y clasificar adecuadamente esta alteración, con el fin de hacer un mejor abordaje y un manejo efectivo del problema. De lograrse estos dos propósitos, la comunicación entre los profesionales de la salud a cargo de pacientes con insuficiencia o falla intestinal puede favorecer un mejor desenlace clínico ${ }^{(2)}$.
La disminución de la función intestinal que no requiere suplementación parenteral se considera una situación de insuficiencia o deficiencia intestinal, tanto para la ESPEN como para la European Society of Intensive Care Medicine (ESICM). A su vez se hace relación a ella como una disfunción gastrointestinal que afecta el cumplimiento de las metas calóricas del paciente, pero sin una indicación clara de cómo avanzar en la progresión del soporte nutricional ${ }^{(3,4)}$.

El objetivo de esta revisión es presentar la relación bidireccional de la insuficiencia y falla intestinal y su influencia en el desarrollo de la malnutrición hospitalaria.

\section{DEFINICIÓN Y CLASIFICACIÓN DE LA FALLA INTESTINAL}

La definición de falla intestinal se ha modificado en dos ocasiones desde que se asumió como una falla orgánica. En 1981, Remington y colaboradores la definieron como "una reducción en el funcionamiento de la masa intestinal por debajo de la cantidad mínima necesaria para una adecuada digestión y absorción de alimentos"(5). En el 2015, ESPEN la modificó para expresar que una situación de falla intestinal requiere la suplementación parenteral de nutrientes, electrólitos o agua.

Existen dos tipos de clasificación de falla intestinal realizados por la ESPEN y ESICM. La primera la clasifica en aguda y crónica, según el tiempo de duración de la misma; esta fue el resultado del trabajo de dos grupos de estudio, uno en 1992, denominado "Grupo de falla intestinal crónica y nutrición artificial en el hogar" y otro el "Grupo de falla intestinal aguda", en 2010. Para esta clasificación, la sociedad tuvo en cuenta los 
Tabla 1. Clasificación de la falla intestinal según la ESPEN. Tomado de Pironi ${ }^{(1)}$

\begin{tabular}{|c|c|l|}
\hline Tipo de falla & Duración & \multicolumn{1}{c|}{ Características } \\
\hline I & Aguda & Corta duración, autolimitada \\
\hline II & Crónica & Requiere suplementación intravenosa por semanas o meses. Pacientes metabólicamente inestables \\
\hline III & Crónica & $\begin{array}{l}\text { Pacientes metabólicamente estables que requieren suplementación intravenosa por meses o años. } \\
\text { Puede o no ser reversible }\end{array}$ \\
\hline
\end{tabular}

Tabla 2. Clasificación de la falla intestinal según la ESICM. Modificado de ${ }^{(1,10)}$

\begin{tabular}{|c|l|}
\hline $\begin{array}{c}\text { Grado de la falla } \\
\text { intestinal }\end{array}$ & \multicolumn{1}{|c|}{ Características } \\
\hline I & $\begin{array}{l}\text { Riesgo. Náuseas o vómitos primeros días posoperatorio, disminución de motilidad intestinal en fase precoz } \\
\text { del shock }\end{array}$ \\
\hline II & $\begin{array}{l}\text { Disfunción gastrointestinal; gastroparesia, con aumento del residuo gástrico. Parálisis del tracto } \\
\text { gastrointestinal, diarrea, aumento de la presión intraabdominal (PIA), sangrado digestivo, intolerancia a la } \\
\text { nutrición enteral, si en } 72 \text { horas no se logra proveer } 20 \text { cal/kg }\end{array}$ \\
\hline II & $\begin{array}{l}\text { Falla intestinal aguda, persistencia de la intolerancia, residuo gástrico aumentado, parálisis, aumento de la } \\
\text { PIA y la presión de perfusión abdominal baja (<60 mm Hg) }\end{array}$ \\
\hline IV & $\begin{array}{l}\text { Falla intestinal aguda. Isquemia intestinal con necrosis, hemorragia gastrointestinal. Shock hemorrágico, } \\
\text { síndrome de Ogilvie, síndrome compartimental abdominal, riesgo vital inmediato }\end{array}$ \\
\hline
\end{tabular}

aspectos funcionales, fisiopatológicos y, para el caso de la falla intestinal crónica, también los clínicos (Tabla 1) $(6,7)$. Mientras tanto, la ESICM establece la clasificación de falla intestinal en cuatro grados, con base en la progresión de la severidad o lesión gastrointestinal, como resultado del trabajo realizado por su grupo de estudio llamado "Grupo de estudio en problemas abdominales” (Tabla 2) $)^{(8,9)}$.

\section{EPIDEMIOLOGÍA}

La incidencia de la falla intestinal aguda según la ESPEN es de 9 pacientes por millón, datos obtenidos a partir de un estudio británico del 2006, mientras que de la falla intestinal crónica es de 7,7 a 15 pacientes por millón. Estos últimos datos se obtuvieron a partir de los registros de nutrición parenteral del 2015 en el hogar de 65 centros de referencia europeos de nutrición parenteral en el hogar en 22 países, y que incluyó a 2919 pacientes, lo cual indica lo poco frecuente que es la falla intestinal ${ }^{(6)}$.

En este grupo de pacientes, el síndrome de intestino corto fue el mecanismo fisiopatológico más frecuente de la falla intestinal crónica $(64,3 \%)$, de los cuales, el $36,8 \%$ tuvo una yeyunostomía terminal, el 19,9\% tenía parte del colon y el 5,9\% todo el colon en continuidad. La dismotilidad intestinal estuvo presente en el 17,5\% de los casos, fístulas en 7,0\%, enfermedad extensa de las mucosas en $6,8 \%$ y obstrucción mecánica en $4,4 \%$. La enfermedad de base más frecuente fue la enfermedad de Crohn $(22,4 \%)$, seguida de isquemia mesentérica $(17,7 \%)$, complicaciones quirúrgicas $(15,8 \%)$, seudoobstrucción intestinal crónica primaria $(9,7 \%)$ y enteritis por radiación (7,3\%). La obtención de este tipo de datos de forma actualizada y por país puede permitir una mejor asignación de recursos a los sistemas de salud para el manejo de estas patologías ${ }^{(6)}$.

\section{DIFERENCIAS DE LAS CLASIFICACIONES DE FALLA INTESTINAL}

$\mathrm{Al}$ analizar las dos clasificaciones descritas se observa que únicamente entre el tipo I de la guía de la ESPEN y el grado I de la guía de la ESCIM existe cierta concordancia en la clasificación de la falla intestinal, al considerar que estos dos tipos son un estado agudo. El tipo II y III de la ESPEN no se corresponden con los grados II a IV de la ESCIM, debido a que en los primeros únicamente se considera el estado crónico de la enfermedad, mientras que en los segundos pueden ser tanto agudos 
como crónicos. Por tanto, la diferencia entre las dos sociedades es el enfoque que cada una le da a la clasificación, mientras la ESPEN prioriza el tiempo de duración de la enfermedad, la ESCIM destaca la severidad de la misma ${ }^{(9)}$.

La clasificación de falla intestinal realizada por la ESPEN y la ESICM no indican cuando al paciente se le debe iniciar la vía oral o enteral. Para pacientes con esta enfermedad, la ESPEN indica que siempre se les debe suministrar soporte nutricional parenteral, mientras que la ESICM lo deja abierto a la interpretación del equipo profesional a cargo del paciente ${ }^{(9)}$, y sugiere como punto de referencia para el cambio en el suministro de la nutrición enteral a parenteral cuando el paciente no tolere las $20 \mathrm{cal} / \mathrm{kg} /$ día durante 3 días consecutivos, acompañado del uso de opioides, procinéticos, y, en general, de un protocolo de manejo clínico, siempre dejando a la interpretación de los síntomas el avance del soporte nutricional ${ }^{(9,11)}$.

Según nuestra opinión, ninguna de las dos clasificaciones ofrece a los grupos de trabajo un abordaje nutricional claro de la situación y, por el contrario, confunden el manejo del soporte nutricional, el cual tiene como finalidad llevar al paciente a condiciones de vida normales, con un tracto gastrointestinal funcional. Por tal motivo, es preciso determinar un enfoque que facilite la toma de decisiones para los grupos de soporte nutricional o profesionales a cargo del paciente con enfermedades que derivan en insuficiencia o falla intestinal $^{(1,12)}$.

De una manera más simple, la mayoría de los pacientes con insuficiencia intestinal en unidades de cuidado crítico se podría incluir en la falla intestinal aguda de tipo I y II según la ESPEN. Para estos tipos de falla intestinal, la vía de alimentación parenteral está indicada, para lo cual es importante advertir que conforme mejora la falla intestinal se puede avanzar la nutrición vía oral o enteral, con indicaciones más precisas para este paso, pudiendo sugerir tipo de fórmulas o alimentos de inicio, osmolaridad, cantidad o dosis a suministrar, y cada cierto tiempo avanzar en las características de preparación o composición de las mismas.

La falta de unificación de los criterios de la definición de la falla intestinal genera que los profesionales de la salud a cargo suplan la información faltante en los manejos indicados en las guías de manera ineficiente. La recomendación de manejo nutricional y médico que dan las diferentes guías internacionales, como la de soporte nutricional parenteral, manejo de fístulas y síndrome de intestino corto, son muy útiles para pato- logías específicas, y han contribuido enormemente a la atención de este tipo de pacientes. Sin embargo, un abordaje de manejo nutricional más enfocado en la funcionalidad gastrointestinal podría permitir visibilizar la falla o insuficiencia intestinal, para poder hacer un manejo integral que permita disminuir la morbimortalidad actual derivada de esta situación ${ }^{(13,14)}$.

El restablecimiento de la funcionalidad perdida es el objetivo del tratamiento, lo que implica hacer un abordaje según las necesidades de cada paciente. Es decir, se debe entender que, dada la complejidad del tracto gastrointestinal, no hay una única forma de manejo nutricional y médico para estas situaciones, ya que frecuentemente derivan en múltiples desenlaces clínicos que cruzan de diferente manera de la insuficiencia a falla intestinal, por lo que se requiere un manejo médico y nutricional específicos ${ }^{(1,15)}$.

El manejo nutricional de la insuficiencia intestinal es poco abordado en ambas sociedades, encontrando indicación médica y nutricional para las situaciones de falla, con uso de nutrición parenteral, y sugiriendo el uso enteral tan pronto como sea posible, sin una indicación clara en el proceso de rehabilitación intestinal para el paso de la falla hacia la insuficiencia intestinal, la cual puede ser una situación tanto desencadenante como ser el desenlace de un proceso crónico. En otras palabras, al referirse del manejo de cualquier tipo de situación intestinal durante la hospitalización de un paciente se debe tener en cuenta la posibilidad de este paso bidireccional ${ }^{(16,17)}$.

\section{LA DOBLE VÍA: DE LA INSUFICIENCIA A LA FALLA INTESTINAL}

La doble vía en la disfunción gastrointestinal implica el paso de la insuficiencia a la falla intestinal, y viceversa. Frecuentemente, algunos signos clínicos, como náuseas, emesis, distensión abdominal, residuo gástrico elevado, presión intraabdominal elevada, dolor abdominal, diarrea, constipación, sangrados digestivos y flatulencia se observan en estas patologías, los cuales para el médico a cargo son vistos, en ocasiones, como signos banales y frecuentes en los pacientes de cuidado crítico.

Según la ESICM, el $60 \%$ de los pacientes tiene al menos uno de estos síntomas por día, sin embargo, no son diagnosticados con insuficiencia ni con falla intestinal. Aunque se reconozca la insuficiencia, esta no se asocia con una posible situación de avance de la misma a falla, y tampoco a la desnutrición generada por la disminución en el aporte nutricional, al que frecuentemente 
son sometidos estos pacientes. A pesar de esto, la presencia de los síntomas de insuficiencia intestinal no implica necesariamente el avance de la misma hacia la falla ${ }^{(18,19)}$.

El paso bidireccional entre la insuficiencia y la falla intestinal requiere un abordaje integral, que permita reconocer los puntos críticos a intervenir para evitar un mayor deterioro del paciente. El tratamiento de la falla intestinal implica un trabajo interdisciplinario, que incluya al cirujano, al intensivista, al gastroenterólogo, al nutricionista, a los enfermeros, al químico farmaceuta, al psicólogo y al trabajador social, entre otros, todos trabajando en pro de la rehabilitación intestinal del paciente hasta alcanzar la alimentación vía oral ${ }^{(20,21)}$.

La falta de reconocimiento de la sintomatología de la insuficiencia intestinal como una condición de prefalla, da origen al manejo médico y nutricional subjeti$\operatorname{vos}^{(22)}$. Lo anterior, sumado a la heterogeneidad de los pacientes, hace que las intervenciones en este sentido se planteen a partir de las experiencias personales de los equipos de salud.

Por otro lado, el manejo objetivo de una enfermedad permite no solo disminuir costos al sistema de salud, al reducir los días de estancia hospitalaria, las infecciones asociadas y el uso de medicamentos, sino reducir el tiempo de rehabilitación intestinal del paciente, mejor preparación para las cirugías de alargamiento intestinal en los casos más complejos y, finalmente, mejorar la calidad de vida del paciente, con un mejor estado nutricional, mejor percepción de su estado de salud y mayor vida útil en los años que esa persona pueda vivir ${ }^{(23,24)}$.

\section{MALNUTRICIÓN INTRAHOSPITALARIA E INSUFICIENCIA O FALLA INTESTINAL}

La prevalencia de la desnutrición intrahospitalaria también afecta a los pacientes con insuficiencia o falla intestinal. Es claro observar que la atención nutricional inadecuada de los pacientes hospitalizados es una de las principales causas para el aumento de la desnutrición en el ámbito hospitalario( ${ }^{(23)}$. Esto, sumado a que un paciente con alteración de su función intestinal presenta un riesgo mayor de desnutrición por la disminución en su capacidad de absorción de nutrientes y riesgo de sepsis por translocación bacteriana, implica que este tipo de pacientes con insuficiencia o falla intestinal presenta mayor riesgo de desnutrición (Figura 1) ${ }^{(25)}$. Es necesario reconsiderar la forma de atención del equipo de salud ante este tipo de paciente, que requiere no solo una rápida atención médica especializada y un nutricionista experto en el área clínica, sino un abordaje integral en su proceso de recuperación, el cual implica fisioterapia, terapia ocupacional, psicología, fonoaudiología y trabajo social, entre otras ${ }^{(26)}$.

Desde 1974, Butherwooth explicó, con el ejemplo del esqueleto en el closet, el problema de la malnutrición como una situación poco visible al interior de los hospitales, con desenlace mortal debido a la falta de un diagnóstico integral ${ }^{(27)}$. Desde entonces son múltiples los estudios realizados que abordan el tema, indicando claramente su prevalencia y la asociación con los factores determinantes para su desarrollo. Observar al esqueleto en el closet no es suficiente, es necesario sacarlo y comunicarse con él. El adecuado abordaje de la problemática nutricional en estos pacientes es la clave para una atención ajustada a las necesidades de cada uno, lo cual, junto con un equipo de salud capacitado en dicho manejo, puede mejorar los desenlaces clínicos de este tipo de patologías, como lo son la insuficiencia y falla intestinal, situaciones que conllevan un mayor riesgo de desnutrición ${ }^{(1,2,28)}$.

A partir de los resultados de estos estudios se desarrollaron estrategias para evidenciar la malnutrición intrahospitalaria. Iniciativas como la del nutrition$\operatorname{Day}^{(28)}$ (https://www.nutritionday.org/), que desde el 2006 mide la malnutrición intrahospitalaria en los países participantes y observa las diferencias en el manejo nutricional de los pacientes, han permitido verificar problemas frecuentes, entre ellos, la ausencia de toma y registro de peso y talla de los pacientes, falta de tamizaje nutricional, baja ingesta sin complementación nutricional oral, ni seguimiento a las metas calóricas y proteicas establecidas, iniciación tardía de la nutrición enteral o parenteral, suspensión de la nutrición por residuos gástricos elevados, emesis, diarreas y distensión abdomi$\mathrm{nal}^{(29,30)}$. Todas estas situaciones adversas no permiten disminuir la prevalencia de la malnutrición hospitalaria, y son de frecuente presentación en los pacientes con insuficiencia y falla intestinal.

Actualmente, The Global Leadership Initiative on Malnutrition (GLIM) propone incluir criterios fenotípicos y etiológicos para el diagnóstico y el grado de desnutrición $^{(31)}$. La presencia de inflamación se ha establecido como un criterio etiológico de la malnutrición, lo cual, junto con la evolución del peso antes del ingreso y durante la estancia en la unidad de cuidados intensivos, puede añadir el factor fenotípico también.

Esta situación es común en los pacientes críticos, y permite confirmar la presencia de desnutrición fácilmente en los pacientes con insuficiencia o falla intestinal, ya que estos siempre tienen pérdidas de peso 


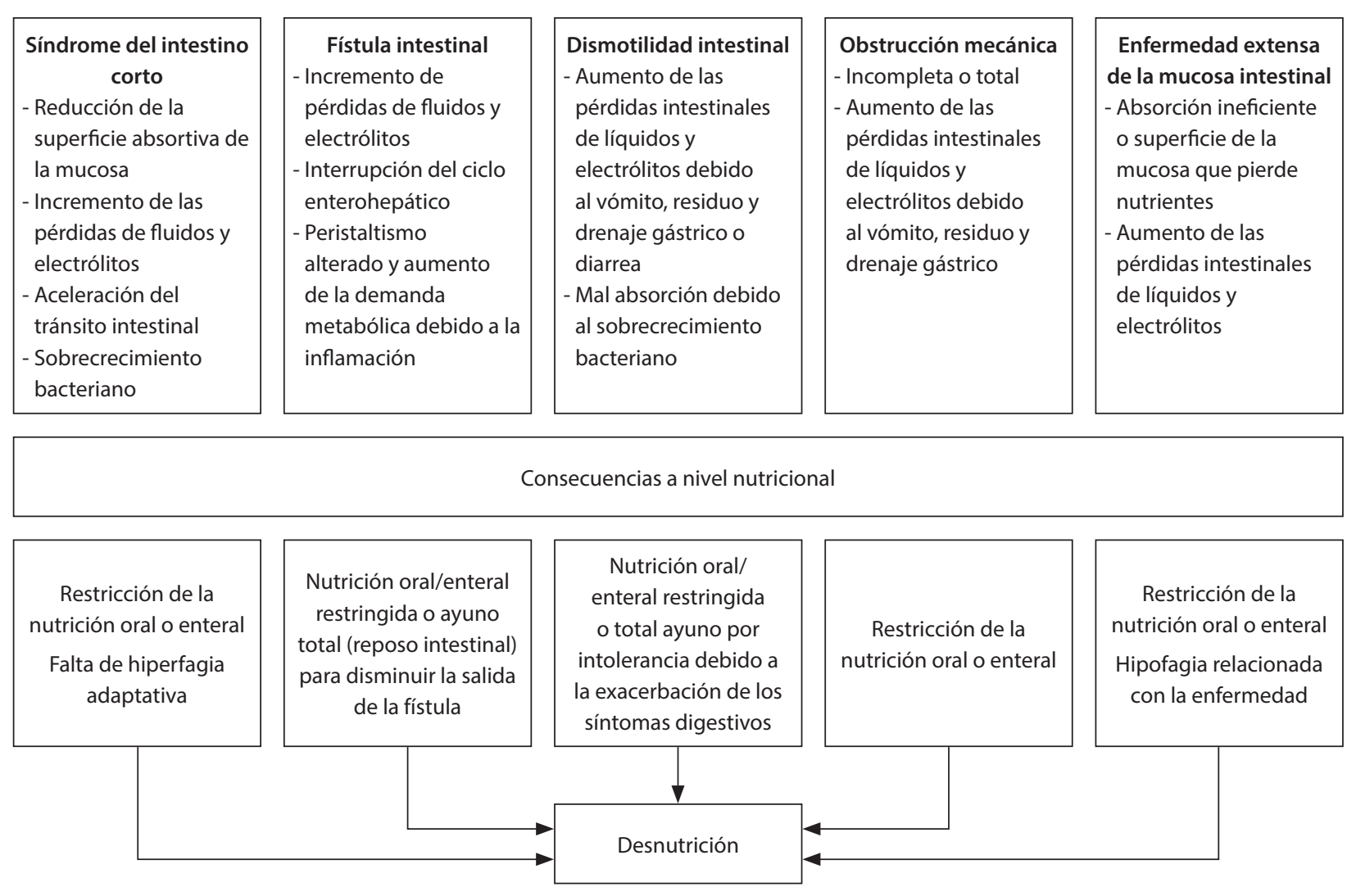

Figura 1. Consecuencias nutricionales de la falla y la insuficiencia intestinal.

durante su hospitalización debido tanto a la presencia de inflamación como a la dificultad de un aporte nutricional pleno, que logre metas calóricas, dada la disfunción gastrointestinal presente.

Autores, como Solar y colaboradores de la Fundación Favaloro de Argentina, compararon el uso de los criterios GLIM con la valoración global subjetiva en pacientes con falla intestinal tipo III, y encontraron que estos pueden ser más efectivos (69,7\% frente a 51,2\%) para evaluar la presencia de desnutrición en este tipo de pacientes, y que a su vez indica que tiene un mayor grado de desnutrición del que se consideraba con otros métodos $^{(32)}$.

No obstante, en el estudio de Kohler y colaboradores se evaluó con los criterios GLIM, la frecuencia y la severidad de la malnutrición en los pacientes con falla intestinal y nutrición parenteral a domicilio e insuficiencia intestinal con nutrición enteral. Los criterios GLIM fueron capaces de identificar, con aproximadamente la misma frecuencia, la malnutrición en la insuficiencia intestinal, pero no en la falla, cuando se combinó la puntuación del pronóstico de Glasgow con el índice de masa corporal (IMC), la masa libre de grasa y la fuerza de agarre. Los autores concluyeron que es necesario validar la utilización de GLIM en estos pacientes ${ }^{(33)}$.

Los centros que cuentan con suministro de nutrición parenteral en el hogar para pacientes con falla intestinal crónica han tenido dificultades en la atención durante la actual de pandemia de COVID-19. Una dificultad importante ha sido el cambio en el seguimiento de rutina de los pacientes, el cual pasó de ser cara a cara a utilizar la telemedicina, con una reducción asociada en el seguimiento de rutina de procedimientos bioquímicos y radiológicos. Estas dificultades se pusieron en evidencia en una encuesta realizada a médicos de 78 centros, con una representación de 3500 pacientes aproximadamente. Se mostró que 37 pacientes con falla intestinal crónica y uso de nutrición parenteral a domicilio tuvieron COVID-19, evidenciando un impacto negativo en los pacientes, debido a la seguridad en términos de provisión de elementos de protección personal, bolsas de nutrición parenteral, personal de enfermería disponible 
y bienestar psicológico, pudiendo afectar su estado nutricional. La opinión de los médicos de cada centro fue que la pandemia estaba teniendo un impacto negativo en la psicología de su paciente en términos de sentimientos, ansiedad, miedo, depresión y pensamientos relacionados con el aislamiento o el confinamiento ${ }^{(34)}$.

La estancia hospitalaria del paciente en estado de desnutrición suele ser más prolongada, y genera altos costos al sistema de salud, ya que frecuentemente se presentan infecciones y complicaciones asociadas con desnutrición y pérdida de su productividad, lo que disminuye la calidad de vida, con efectos negativos sobre el entorno familiar y social ${ }^{(35,36)}$. Esto se observó por autores como Enríquez y colaboradores, en un estudio retrospectivo realizado en el hospital central de Chihuahua, México, en 53 pacientes ingresados en una unidad de falla intestinal, donde se encontró que estas patologías tienen estancias muy prolongadas, con un promedio de 67,7 días, y una asociación entre el tipo de falla intestinal y la estancia hospitalaria, lo que aumentó la morbilidad y la mortalidad, así como los costos y los efectos secundarios asociados. En este mismo estudio se menciona que la presencia de enfermedad diarreica aguda prolongada, tanto en pacientes sometidos a procedimientos quirúrgicos como aquellos que no, aumenta la estancia hospitalaria, las infecciones y la mortalidad a 30 días. A su vez, la presencia de íleo posoperatorio incrementa tanto la estancia hospitalaria como las complicaciones posoperatorias, reintervenciones, reingresos y mortalidad, en comparación con aquellos pacientes en los que se recuperó la función intestinal temprana, lo cual generó un mayor costo en la atención. En este mismo estudio se evaluó la formación del personal hospitalario en el manejo de estos pacientes, encontrando que quienes desconocían el abordaje multidisciplinar del paciente con falla intestinal y la estandarización del manejo del mismo llevó a que la evolución de estos pacientes no fuera la esperada y tuvieran estancias más prolongadas; sin embargo, a medida que los equipos se familiarizaron con el manejo de pacientes con esta patología se observó una disminución en la estancia ${ }^{(37)}$.

De manera similar, en otro estudio retrospectivo con 331 pacientes, realizado por el departamento de Gastroenterología del Hospital Rigs en Copenhague, Dinamarca, se encontró que los pacientes con falla intestinal crónica tienen mayores días de hospitalización asociados con mayor tiempo de uso de la nutrición parenteral en el hogar, con un promedio 1,7 años de uso de nutrición parenteral, de los cuales, el 6,6 \% de este tiempo requirió de hospitalización, con un promedio de estancia de 7 días por cada hospitalización requerida, lo que evidencia un mayor costo en la atención de este tipo de pacientes ${ }^{(38)}$. Este estudio también sugiere cómo en algunos casos se puede disminuir la necesidad de soporte nutricional parenteral por medio de la hiperfagia, como mecanismo que promueve la adaptación intestinal endógena e innata, y proporciona una estrategia óptima para una adaptación intestinal espontánea, para lo cual es importante realizar una correcta atención nutricional, que posibilite un abordaje oportuno, efectivo y adaptado a las necesidades individuales del paciente, en pro de una recuperación integral ${ }^{(38)}$.

Otro estudio de cohorte que incluyó a 77 pacientes con falla intestinal crónica y el uso de nutrición parenteral en el hogar, realizado en un centro de referencia de insuficiencia intestinal del Centro para Nutrición y Enfermedad Intestinal (CET) del Hospital Universitario de Aalborg, Dinamarca, evaluó datos de antropometría, composición corporal valorada por impedancia bioeléctrica y fuerza muscular por fuerza de agarre manual, número de readmisiones hospitalarias anuales y duración de la estancia hospitalaria, al igual que la mortalidad, encontrando que el ángulo de fase de manera independiente predijo los reingresos y la duración de la estancia hospitalaria $(p=0,009)$, mientras que la fuerza de agarre de la mano se asoció con el número de reingresos $(p=0,012)$.

Por otro lado, el ángulo de fase y el índice de masa libre de grasa se asociaron con la supervivencia, con una mortalidad 4 veces mayor en pacientes con índice de masa libre de grasa baja y una mortalidad 5 veces mayor en pacientes con un bajo ángulo de fase. Estos hallazgos subrayan la importancia de enfocarse en la terapia nutricional junto con la actividad física, con el fin de mejorar el resultado en pacientes metabólicamente estables con falla intestinal crónica, como parte del abordaje integral, que permite disminuir costos por reingresos y estancias hospitalarias prolongadas ${ }^{(39)}$.

En un estudio de dos casos, la nutrición parenteral domiciliaria supervisada por enfermeras mostró buenos resultados al mejorar la valoración global subjetiva como medición del estado nutricional de los pacientes, pasando de la calificación $\mathrm{C}$ a $\mathrm{B}$ en 3 meses, cuando los pacientes se sometieron a reanastomosis yeyunal y regresaron a casa 1 semana después sin complicaciones. En el seguimiento posoperatorio al mes y al año, ninguno de los pacientes presentó complicaciones. Los pacientes 
tampoco experimentaron complicaciones de filtración o infección posoperatoria, mientras que la función digestiva también se recuperó. Este método puede considerarse como terapia complementaria para la insuficiencia intestinal traumática de alto gasto antes de someterse a una nueva anastomosis, lo que puede disminuir costos en cuanto a reingresos, estancias hospitalarias y, en gene$\mathrm{ral}$, al sistema de salud y pérdida de calidad de vida ${ }^{(40)}$.

De igual forma, como se plantea en los estudios abordados, para una atención más oportuna y completa es preciso desarrollar investigaciones que permiten conocer la incidencia y prevalencia de este tipo de patologías en cada país, y así proporcionar herramientas a las personas que toman decisiones en temas de salud pública, sobre cómo enfrentar tanto la insuficiencia como la falla intestinal, en el ámbito de cuidado crítico, hospitalización y cuidado domiciliario.

\section{CONCLUSIONES}

La insuficiencia y falla intestinal son situaciones que pueden generarse de manera bidireccional. Las diferencias en la clasificación de la falla intestinal, según las diversas asociaciones, afectan el manejo de los pacientes; por tanto, es preciso ajustar las prácticas a un enfoque más simple e integral, que permita el abordaje desde el punto de vista médico y nutricional. Del mismo modo, la malnutrición por pérdida de peso rápida es muy común en el paciente crítico. La presencia de insuficiencia o falla intestinal en estos pacientes aumenta la probabilidad de su ocurrencia, toda vez que la absorción de nutrientes se ve disminuida y, por ende, los hace más vulnerables a desarrollar una mayor pérdida de peso, lo que aumenta su morbimortalidad.

\section{PUNTOS CLAVE}

Insuficiencia y falla intestinal como desencadenantes de la desnutrición:

- La insuficiencia intestinal como desencadenante de la falla intestinal.

- Clasificación de la falla intestinal según dos asociaciones internacionales.

\section{Agradecimientos}

El desarrollo del presente artículo contó con el apoyo del grupo de investigación en nutrición y metabolismo de la Universidad de Caldas, al cual queremos agradecerles.

\section{Financiamiento}

Para el desarrollo del presente artículo la financiación empleada fue de fuentes propias de los autores. El presente estudio no tuvo financiación de otras fuentes.

\section{Conflictos de interés}

Los autores manifestamos no tener ningún tipo de conflicto de interés, económico, profesional o personal para el desarrollo de este artículo. Además de haber participado en la concepción y realización del trabajo que dio origen al documento, de haber participado en la redacción del texto y sus revisiones y de autorizar la publicación de la versión final.

\section{Declaración de autoría}

DTG, CHGC, WNS contribuyeron igualmente a la concepción y diseño de la investigación; CHGC apoyó al diseño de la investigación; DTG contribuyó a la adquisición y análisis de los datos; DTG, CHGC y WNS aportaron en la interpretación de los datos y redactaron el manuscrito. Todos los autores revisaron el manuscrito, acuerdan ser plenamente responsables de garantizar la integridad y precisión del trabajo, y leyeron y aprobaron el manuscrito final.

\section{Referencias bibliográficas}

1. Pironi L, Arends J, Bozzetti F, Cuerda C, Gillanders L, Jeppesen PB, et al. ESPEN guidelines on chronic intestinal failure in adults. Clin Nutr. 2016;35(2):247-307. doi: 10.1016/j.clnu.2016.01.020.

2. Nightingale JMD, Small M, Jeejeebhoy K. Intestinal failure definition and classification comments: Good in parts but could be better. Clin Nutr. 2016;35(2):536. doi: 10.1016/j. clnu.2015.10.013.

3. Pironi L, Arends J, Baxter J, Bozzetti F, Peláez RB, Cuerda $C$, et al. ESPEN endorsed recommendations. Definition and classification of intestinal failure in adults. Clin Nutr. 2015;34(2):171-80. doi: 10.1016/j.clnu.2014.08.017.

4. Prahm AP, Brandt CF, Askov-Hansen C, Mortensen PB, Jeppesen $\mathrm{PB}$. The use of metabolic balance studies in the objective discrimination between intestinal insufficiency and intestinal failure. Am J Clin Nutr. 2017;106(3):831-38. doi: 10.3945/ajcn.117.158386.

5. Remington MM, Fleming CR, Zinsmeister AR, Malagelada, JR. Gastrointestinal motility patterns in the short bowel syndrome (SBS): Effect of a synthetic opiate. Gastroenterology. $1981 ; 80(5): 1260$. 
6. Pironi L, Corcos O, Forbes A, Holst M, Joly F, Jonkers C, et al. Intestinal failure in adults: Recommendations from the ESPEN expert groups. Clin Nutr. 2018;37(6):1798-809. doi: 10.1016/j.clnu.2018.07.036.

7. Kappus M, Diamond S, Hurt RT, Martindale R. Intestinal failure: New definition and clinical implications. Curr Gastroenterol Rep. 2016;18(9):48. doi: 10.1007/s11894016-0525-x.

8. Brandt CF, Tribler S, Hvistendahl M, Staun M, Brøbech P, Jeppesen PB. Single-center, adult chronic intestinal failure cohort analyzed according to the ESPEN-endorsed recommendations, definitions, and classifications. JPEN J Parenter Enteral Nutr. 2017;41(4):566-74. doi: 10.1177/0148607115612040.

9. Reintam Blaser A, Malbrain MLNG, Starkopf J, Fruhwald S, Jakob SM, De Waele J, et al. Gastrointestinal function in intensive care patients: Terminology, definitions and management. Recommendations of the ESICM Working Group on Abdominal Problems. Intensive Care Med. 2012;38(3):38494. doi: 10.1007/s00134-011-2459-y.

10. Pironi L, Konrad D, Brandt C, Joly F, Wanten G, Agostini F, et al. Clinical classification of adult patients with chronic intestinal failure due to benign disease: An international multicenter cross-sectional survey. Clin Nutr. 2018;37(2):728-38. doi: 10.1016/j.clnu.2017.04.013.

11. Brandt CF, Hvistendahl M, Naimi RM, Tribler S, Staun M, Brøbech $\mathrm{P}$, et al. Home parenteral nutrition in adult patients with chronic intestinal failure: The evolution over 4 decades in a tertiary referral center. JPEN J Parenter Enteral Nutr. 2017;41(7):1178-87. doi: 10.1177/0148607116655449.

12. Pironi L, Shaffer J; Home Artificial Nutrition \& Chronic Intestinal Failure and the Acute Intestinal Failure Special Interest Groups of ESPEN. Reply to Letter to the Editor Intestinal failure definition and classification comments. Clin Nutr. 2016;35(3):763. doi: 10.1016/j.clnu.2016.03.022.

13. Geoghegan AR, Donohoe CL, Reynolds JV. Acute intestinal failure in surgical patients: An audit of incidence, management and outcomes in an Irish hospital, and compliance with ASGBI guidelines. Ir J Med Sci. 2012;181(4):467-71. doi: 10.1007/s11845-011-0800-7.

14. González-Salazar LE, Guevara-Cruz M, Serralde-Zúñiga AE. Medical and nutritional treatment in adult patients with acute intestinal failure. Rev Clin Esp. 2019;219(3):151-60. doi: 10.1016/j.rce.2018.08.003.

15. Fernández-Ortega JF, Herrero MJI, Martínez GP, Spanish Society of Intensive Care Medicine and Coronary UnitsSpanish Society of Parenteral and Enteral Nutrition (SEMICYUC-SENPE). Guidelines for specialized nutritional and metabolic support in the critically ill-patient. Update. Consensus of the Spanish Society of Intensive Care Medicine and Coronary Units- Spanish Society of Parenteral and Enteral Nutrition (SEMICYUC-SENPE): indications, timing and routes of nutrient delivery. Med Intensiva. 2011;35(1):711. doi: 10.1016/S0210-5691(11)70002-X.
16. Cohran VC, Prozialeck JD, Cole CR. Redefining short bowel syndrome in the 21st century. Pediatr Res. 2017;81(4):540-9. doi: 10.1038/pr.2016.265.

17. Compton F, Bojarski C, Siegmund B, van der Giet M. Use of a nutrition support protocol to increase enteral nutrition delivery in critically ill patients. Am J Crit Care. 2014;23(5):396403. doi: 10.4037 /ajcc2014140.

18. Javid PJ, Wendel D, Horslen SP. Organization and outcomes of multidisciplinary intestinal failure teams. Semin Pediatr Surg. 2018;27(4):218-22. doi: 10.1053/j.sempedsurg.2018.07.005.

19. Mazuski JE. Feeding the injured intestine: Enteral nutrition in the critically ill patient. Curr Opin Crit Care. 2008;14(4):4327. doi: 10.1097/MCC.0b013e328307390b.

20. Merras-Salmio L, Pakarinen MP. Refined multidisciplinary protocol-based approach to short bowel syndrome improves outcomes. J Pediatr Gastroenterol Nutr. 2015;61(1):24-9. doi: 10.1097/MPG.0000000000000775.

21. Oterdoom LH, Ten DSM, de Groot SDW, Arjaans W, van Bodegraven AA. Limited long-term survival after in-hospital intestinal failure requiring total parenteral nutrition. Am J Clin Nutr. 2014;100(4):1102-7. doi: 10.3945/ajcn.114.087015.

22. Pironi L, Paganelli F, Labate AMM, Merli C, Guidetti C, Spinucci G, et al. Safety and efficacy of home parenteral nutrition for chronic intestinal failure: a 16-year experience at a single centre. Dig Liver Dis. 2003;35(5):314-24. doi: 10.1016/ s1590-8658(03)00074-4.

23. Sigalet D, Boctor D, Brindle M, Lam V, Robertson M. Elements of successful intestinal rehabilitation. J Pediatr Surg. 2011;46(1):150-6. doi: 10.1016/j.jpedsurg.2010.09.083.

24. Stefaniak J, Baron DM, Metnitz PGH, Kramer L. Gastrointestinale motilitätsstörungen auf der intensivstation - Ursachen, konsequenzen und therapie. Anasthesiol Intensivmed Notfallmed Schmerzther. 2010;45(11-12):696706. doi: $10.1055 / \mathrm{s}-0030-1268871$.

25. Iapichino G, Callegari ML, Marzorati S, Cigada M, Corbella D, Ferrari S, et al. Impact of antibiotics on the gut microbiota of critically ill patients. J Med Microbiol. 2008;57(8):100714. doi: 10.1099/jmm.0.47387-0.

26. Allan P, Lal S. Intestinal failure: a review. F1000Res. 2018; 7:85. doi: 10.12688/f1000research.12493.1.

27. Butterworth CE. The skeleton in the hospital closet. Nutrition Today. , 1974;9(2):4-8.

28. Hiesmayr M, Schindler K, Pernicka E, Schuh C, SchoenigerHekele A, Bauer P, et al. Decreased food intake is a risk factor for mortality in hospitalised patients: the NutritionDay survey 2006. Clin Nutr. 2009;28(5):484-91.

29. Hiesmayr M, Tarantino S, Moick S, Laviano A, Sulz I, Mouhieddine M, et al. Hospital malnutrition, a call for political action: A public health and nutritionDay perspective. J Clin Med. 2019;8(12):2048. doi: 10.3390/jcm8122048.

30. Cardenas D, Bermudez C, Pérez A, Diaz G, Cortes LY, Contreras $\mathrm{CP}$, et al. Nutritional risk is associated with an increase of in-hospital mortality and a reduction of being dis- 
charged home: Results of the 2009-2015 nutritionDay survey. Clin Nutr. 2020;38:138-45. doi: https://doi.org/10.1016/j. clnesp.2020.05.014.

31. Cederholm T, Jensen GL, Correia MITD, Gonzalez MC, Fukushima R, Higashiguchi T, et al. GLIM criteria for the diagnosis of malnutrition - A consensus report from the global clinical nutrition community. Clin Nutr. 2019;38(1):1-9. doi: 10.1016/j.clnu.2018.08.002.

32. Solar H, Ortega ML, Doeyo M, Crivelli A, Gondolesi G. Intestinal failure type III. Are GLIM criteria better than subjective global assessment to diagnose nutritional status? Clin Nutr. 2020;40:605. doi: 10.1016/j.clnesp.2020.09.597.

33. Køhler M, Mikkelsen S, Østergaard T, Rasmussen HH. Diagnosis of malnutrition in patients with intestinal insufficiency or failure using GLIM-criteria. Clin Nutr. 2020;40:447. doi: 10.1016/j.clnesp.2020.09.126.

34. Allan PJ, Pironi L, Joly F, Lal S, Van Gossum A, Home Artificial Nutrition \& Chronic Intestinal Failure special interest group of ESPEN. An international survey of clinicians> experience caring for patients receiving home parenteral nutrition for chronic intestinal failure during the COVID-19 pandemic. JPEN J Parenter Enteral Nutr. 2021;45(1):43-9. doi: 10.1002/jpen.2050.

35. Ricciardi R, Roberts PL, Read TE, Hall JF, Marcello PW, Schoetz DJ. Which adverse events are associated with mortality and prolonged length of stay following colorectal surgery?
J Gastrointest Surg. 2013;17(8):1485-93. doi: 10.1007/ s11605-013-2224-3.

36. Karagozian R, Johannes RS, Sun X, Burakoff R. Increased mortality and length of stay among patients with inflammatory bowel disease and hospital-acquired infections. Clin Gastroenterol Hepatol. 2010;8(11):961-5. doi: 10.1016/j. cgh.2010.07.017.

37. Enriquez-Sánchez LB, Carrillo-Gorena MJ, Granados-Aldaz LA, Balderrama-Miramontes LF, Gallegos-Portillo LG, RezaLeal CN, et al. Intestinal failure functional classification type associated with an extended length of stay at the intestinal failure unit, Central Hospital in Chihuahua, Mexico. Cir. 2019;87(5):559-63. doi: 10.24875/CIRU.19000767.

38. Fuglsang KA, Brandt CF, Scheike T, Jeppesen PB. Hospitalizations in patients with nonmalignant short-bowel syndrome receiving home parenteral support. Nutr Clin Pract. 2020;35(5):894-902. doi: 10.1002/ncp.10471.

39. Køhler M, Olesen SS, Rasmussen HH. Body composition predicts clinical outcome in patients with intestinal failure on long-term home parenteral nutrition. Clin Nutr ESPEN. 2018;28:193-200. doi: 10.1016/j.clnesp.2018.08.004.

40. Yuda HA, Werdana VAP, Fauzi AR. Nurse supervised combined refeeding and home parenteral nutrition in traumatic intestinal failure: A case series. Int J Surg Case Rep. 2019;61:199-201. doi: 10.1016/j.ijscr.2019.07.049. 